To be published in 4 th Annual IISSC, New Orleans, March, 3992.

CONE $-920331--53$

DE92 018768

\title{
A NOVEL PHOTOMUITIPLIER TUBE FOR CALORIMETRY AT THE SSC
}

P. Uushman', M.Iosue ${ }^{2}$, B.Johnson ${ }^{2}$, M.Madden ${ }^{3}$, R.Rusack 4 ,

M.Szawlowski ${ }^{3}$

YYale University, New Haven, CT

${ }^{2}$ Litton Electron Devices, Tempe, AZ

${ }^{3}$ Advanced Photonix Inc., Los Angeles, CA

${ }^{4}$ Flockefeller University, New York, NY

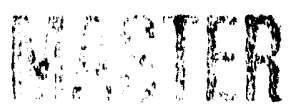

\section{INTRODUCTION}

A silicon target photomultiplier tube with an anode consisting of a large area Avalanche Photodiode (APD) has been developed. The tube combines the advantages of an avalanche photodiode, excellent linearity and large dynamir range, with the properties of a phototube. The tube structure is proximity focussing, which is not affected by high magnetic fields approximately aligned with the axis. The high linearity makes this device attractive for calorimetry at the SSC. The extension of this concept to an APD array will provide a cost effective fiber readout for both digital (tracking) and analogue applications. A tube equipped with an APD array is being developed currently as a readout option for the SDC Shower Maximum Detector.

\section{HYBRID PHOTOTUBE DESIGN}

A $16 \mathrm{~mm}$ diameter APD is located at the anode of a proximity focussed phototube, $6.8 \mathrm{~mm}$ from a photocathode. Fig 1 is a schematic of the tube design. The photocathode is maintained at a negative high voltage ( $8-12 \mathrm{kV})$, the front surface of the APD is held to ground through a $1 \mathrm{k} \Omega$ resistor, and the back surface or anode of the APD is biased with a positive roltage of $1.2 \mathrm{kV}$. Charge is supplied to the APD anode from a $10 \mathrm{nF}$ storage-capacitor to ground and the signal is picied of the APD cathode. No divider chain is required.

The APD is operated in electron bombardment mode: photoelectrons are accelerated by the electrostatic field and strike the APD with energies in the keV range. The gain of this first stage is therefore approximated by the photocathode voltage divided by $3.57 \mathrm{eV}$, once losses at the APD's surface are accounted for. An additional $g$ ain of 200-1000 is provided by the APD itself, yielding an overall tube gain of $5 \times 10^{3}$. Because of the first stage gain, the signal to noise of the hybrid tube is greatly improved over that of a bare APD operating in the optical mode. The second stage of amplification is not present in hybrid tubes equipped with a PIN diode targer, where gains beyond 5000 are dificult to obtain. 


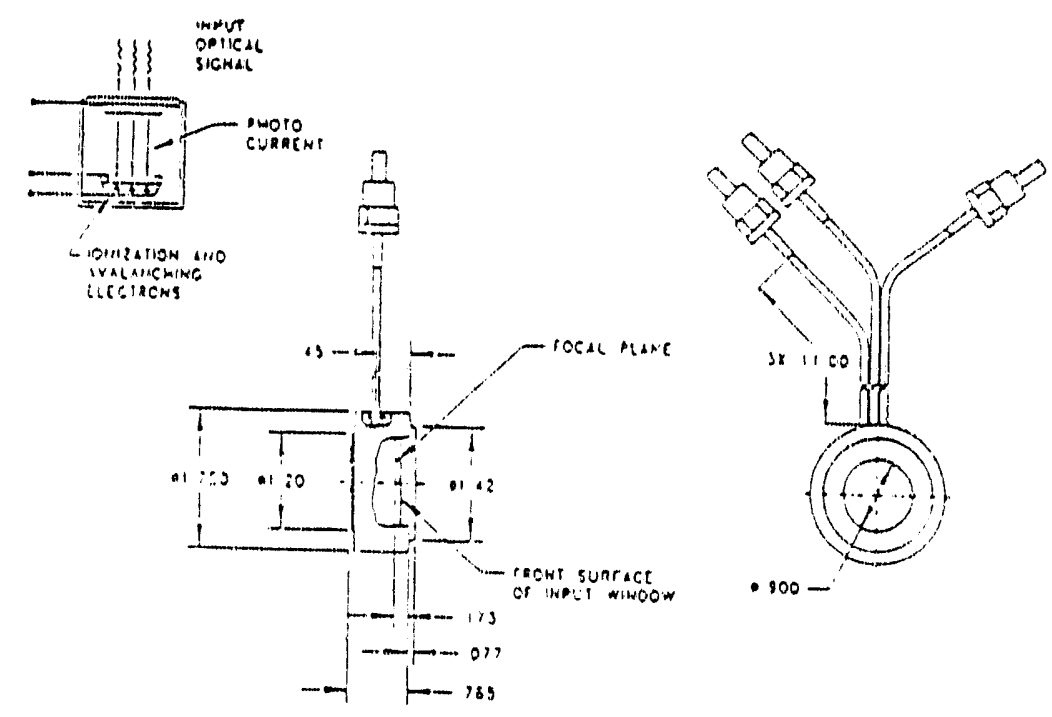

Figure 1: Schematic of tube design and actual prototype dimensions

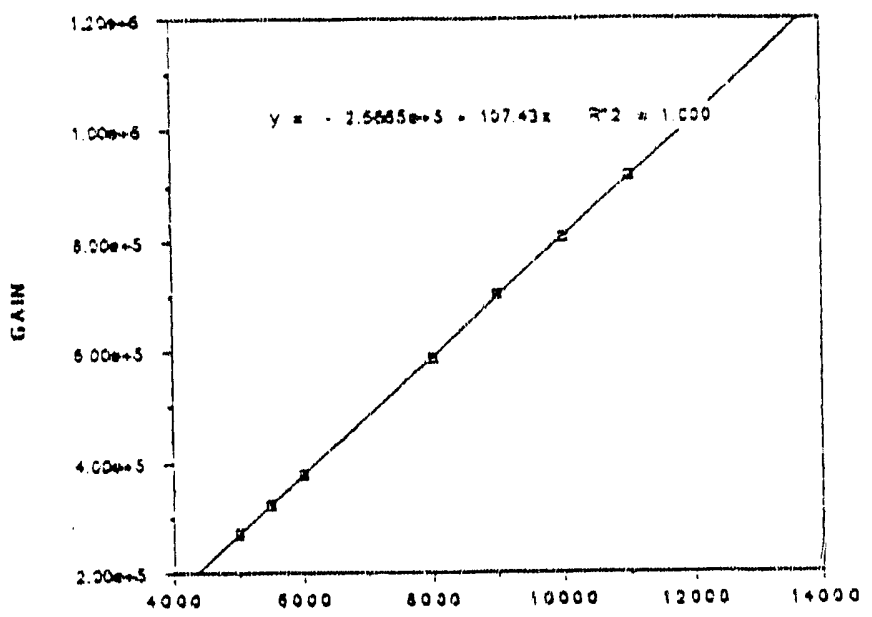

Figure 2: Gain as a function of photocatbode voltage for an APD bias voltage of $2 \mathrm{kV}$.

The characteristic gain curves bave been measured in both pulsed and DC mode. In pulsed mode a scintillator with a spectral peak at $430 \mathrm{~nm}$ was activated by a $\mathrm{N}_{2}$ ultraviolet laser. These curves as a function of both APD reverse bias and gap voltage are shown in fig 2 and 3 . The gain is extremely linear with applied gap voltage $\left(V_{p c}\right)$ above $1000 \mathrm{~V}$, a threshold which depends on the surface treatment of the APD. The gain as a function of reverse bias voltage has a sharp upturn as it approaches the breakdown voltage. The signal to noise also degrades in this region and optimum performance is found at reverse biases $<2000$ volts.

\section{MAGNETIC FDELD RESISTANCE}

Since photoelectrons are accelerated by an electrostatic field, it is easy to calculate the effect of a magnetic field on the tube. To first order, an axial B-field will have no effect on the gain and serve rnainly to constrain the electrons along the field lines. This would be an advantage in segmented anode configurations such as fiber readout since it would minimize optical crosstalk between neighboring cells. As the angle $\theta$ between the B-field axis and the tube (or electrostatic) axis is increased, the iñase is shifted to the side by $\Lambda$ and the gain goes down as the area $A$ of overlap 


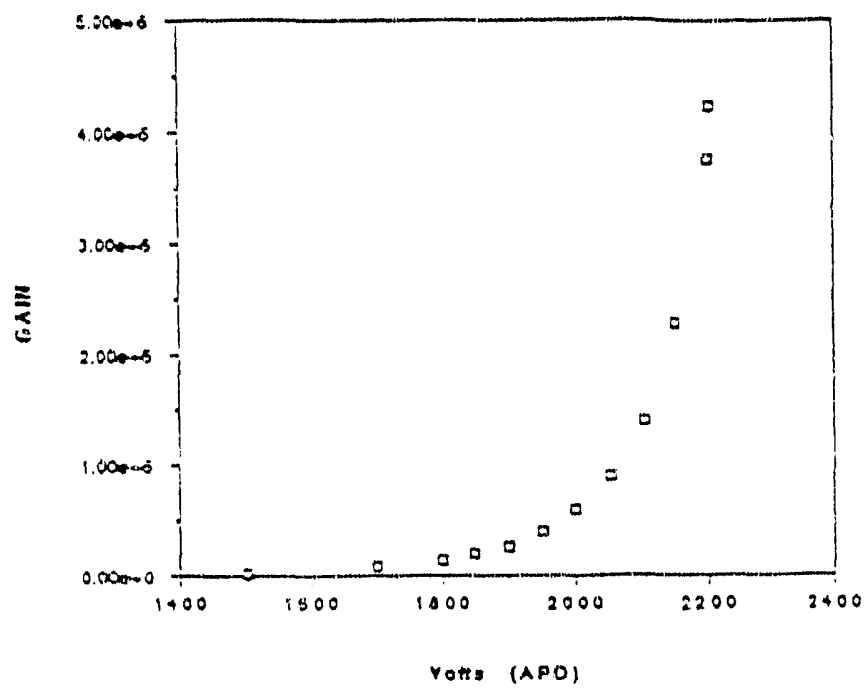

Figure 3: Gain as a function of APD bias voltage for a photocathode voltage of $-8 \mathrm{kV}$.

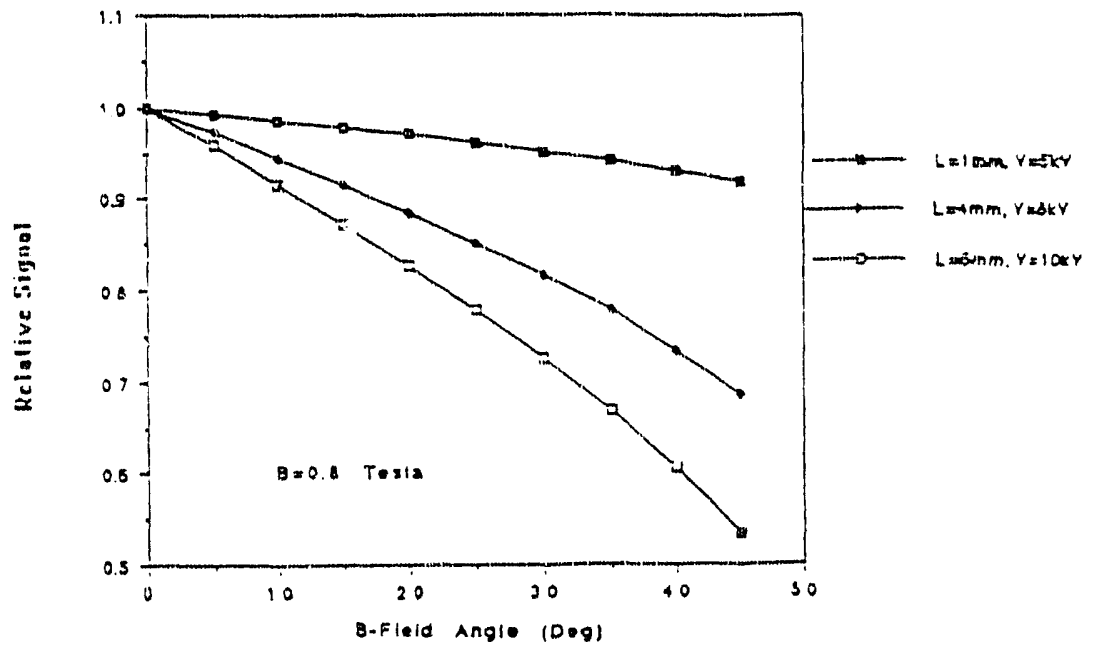

Figure 4: The relative loss in current gain due to an external B-field as a function of the angle between tube and B-field axis. Three different tube geometries are considered.

between displaced image and APD active area:

$$
\frac{i}{i_{0}}=\frac{A}{\pi r^{2}}=\frac{2}{\pi}(\alpha-\sin \alpha \cos \alpha)
$$

where $\alpha=\cos ^{-1}[\Delta / 2 r]$ and $\Delta \approx L / \tan \theta$. This is plotted in fig 4 for three different gap dimensions. Gain would be reduced by roughly $50 \%$ for a B-field at $45^{\circ}$ to the tube axis for the prototype tube design, but this gap can be reduced and depends on which operating voltage (and thus gain) is desired. The $1 \mathrm{~mm} g a p$, however, is probably only practical for photocatbodes with large work functions, such as $\mathrm{CsTe}$ $(\phi=4 \mathrm{eV})$ for which dark currents due to field emission are reduced.

\section{PHOTOCATHODE}

The prototype tube has an S20 photocathode with a radiant response of only $10 \mathrm{~mA} / \mathrm{W}$ at $430 \mathrm{~mm}$ (this was a mistake during processing and is not typical). Other photocathodes are also possible. Tubes with a Gen III, GaAsAl, photocathode are being manufactured in order to improve the sensitivity to scintillators in the longer wavelength, more radiation sesistant regime and to study the materials compatability with III-IV materials. By extending the blue sensitivity of the GaAs-based photocathode, we expect a quantum efficiency of $30 \%$ for green fibers. The response curves for this ano other available photocathodes are shown in fig 5 . 


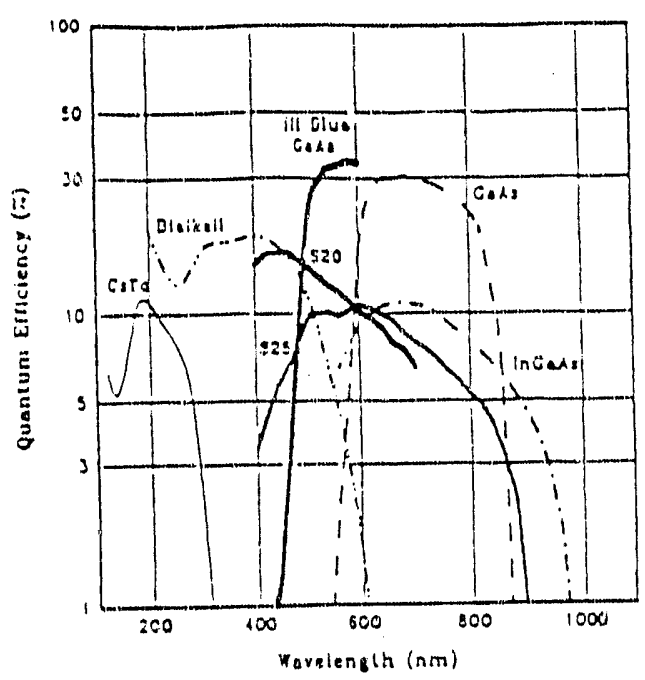

Figure 5: Photocathodes readily available from Litton and their spectral response curves.

Another interesting application of the hybrid tube is ultra-fast calorimetry using $\mathrm{BaF}_{2}$ which requires sensitivity in the ultraviolet. In addition to conventional $\mathrm{CsTe}$ and $\mathrm{CsTeRb}$, Litton is also developing a solid state photocathode, Aluminum Gallium Nitride, which is sensitive to the UV and has 5 orders of magnitude falloff over $80 \mathrm{~nm}$ above $290 \mathrm{~nm}$. This would provide sensitivity to the $B a F_{2}$ fast components at $190 \mathrm{~nm}$ and $220 \mathrm{~nm}$, while suppressing the slow component at $310 \mathrm{~nm}$ by $3 \times 10^{4}$.

\section{APD DESIGN}

These are large area APD's with a "bevelled edge" design. The breakdown voltage is very high with a wide depletion zone. Physically bevelling the edges prevents breakdown at the junction periphery. These APD's therefore have very differen: characteristics than the smaller ones $(50.500 \mu \mathrm{m}$ diam) typically associated with fibe: tracking readout which are produced using the "reach-through" structure.

The ratio of hole to electron ionization rates is

$$
k_{0}=\int_{x_{1}}^{x_{1}} \beta d x / \int_{x_{1}}^{x_{2}} \alpha d x
$$

where the ionization coefficients of electrons and holes respectively are $\alpha$ and $\beta$. This is an important quantity since the lower $k_{0}$, the larger the applied voltage (and thus gain) before the multiplication curve bends to infinity. Fig 3 shows this happening at about 2200 volts for the APD in our prototype tube. Typical $k$-values for the large area bevelled edge APD's are $k \sim 0.0025$, a factor of 10 smaller than typical reach. through designs, due to their lower peak electric field at breakdown ${ }^{1}$ (remember that $\beta / \alpha$ increases with the internal electric field).

Other consequences of a lower $k$-value are lower excess noise factor and less variation in gain due to nonuniformities in the doping level of the depletion layer. The dark currents measured on the set of 8 APD's delivered to Litton for potting ranged from 160-350 $\mathrm{nA}$ for $16 \mathrm{~mm}$ diameter devices at room temperature. After potting, the prototypes had dark currents of $370 \mathrm{aA}$ and $700 \mathrm{nA}$, representing a factor of 2 increase after bake-out over the bare APD dark current.

\section{TIMING CHARACTERISTICS}

A large area also implies large capacitance; $120 \mathrm{pF}$ is typical for the APD in question, which results in a large RC constant and thus slow risetime. The 8 APD's had measured risetimes of $15-40$ ns. However, for the application in mind: Iiver IEâdiot of the SDC Shower Maximum Detector, these large area AFD's will be 


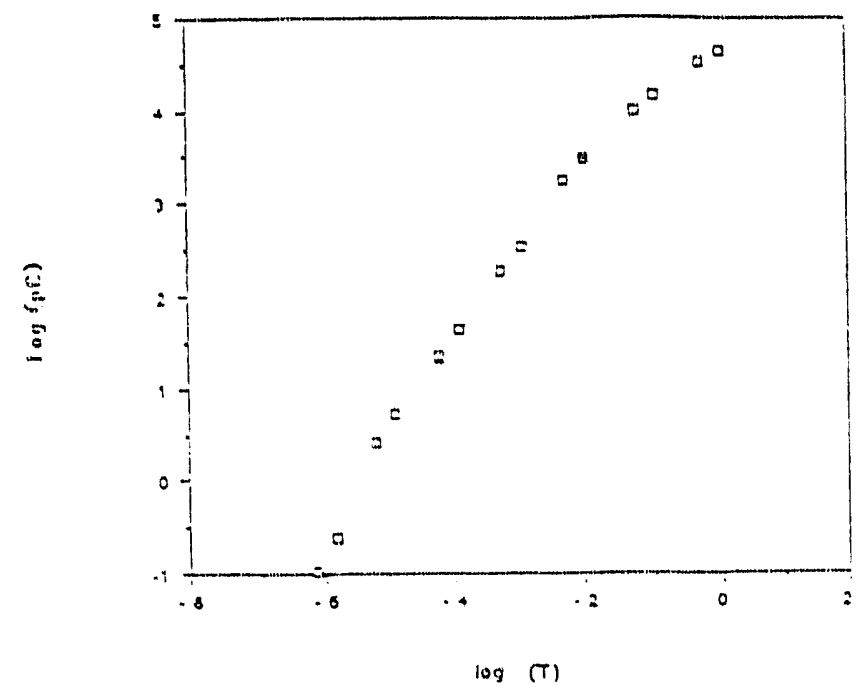

Figure 6: The output charge in picoCoulombs as a function of relative light intensity as defined by the transmission of calibrated neutral density filters.

subdivided into $8 \times 8$ arrays, reducing the capacitance by a factor of 64 such that it no longer influences the risetime, which should then be in the neighborhood of $1-2$ ns.

In either case, the transit time jitter is very small (tens of picoseconds), making these excellent time-of-llight counters. ${ }^{2}$ This is due to 2 factors: (1) since a proximity focussed tube is simply a planar accelerator, the photoelectrons will not travel different path lengths to the collection surace, and (2) the electron-hole pairs are all produced at the surface of the APD by photoelectrons, rather than throughout the device by impinging photons.

\section{TEMPERATURE DEPENDENCE AND UNIFORMITY}

Both the Temperature characteristics and uniformity depend on the APD. The gain of an. APD decreases with increased temperature. A $40 \%$ reduction in gain was observed for a $20^{\circ} \mathrm{C}$ change in temperature from $0^{\circ} \mathrm{C}$ to room temperature. However, since the tube does not need a powered divider chain, it does not heat up during use, making temperature regulation relatively easy. It also introduces the possibility of runring the device at liquid nitrogen temperatures to increase gain and reduce noise.

Nonuniformities in the APD are measured at the $5 \%$ level. Since most nonuriformities in conventional phototubes are due to varying collection efficiencies on the multiplying structures, and since the photocathode itself is very uniform, it is expected that the tube will have less than $10 \%$ variation over the active area. This is a very important property for multi-anode versions of the tube, since it means channelto-channel variations will be negligible. Compare this with the $5: 1$ average variation in channel gain from the test multi-anode phototubes, which puts severe constraints on the digitizing and trigger electronics which follow.

\section{DYNAMIC RANGE AND NOISE}

Measurements of the linearity of this device over 6 orders of magnitude have been performed in both $D C$ and pulsed mode. In the DC mode, a constant light source illuminated the photocathode and the output current was measured directly using an electrometer. No departure from linearity was observed over 6 orders of magnitude. In the pulsed mode (see fig 6), blue light from a scintillator activated by a $N_{2}$ laser neutral density filters reduced the amount of primary light reaching the tube. At 


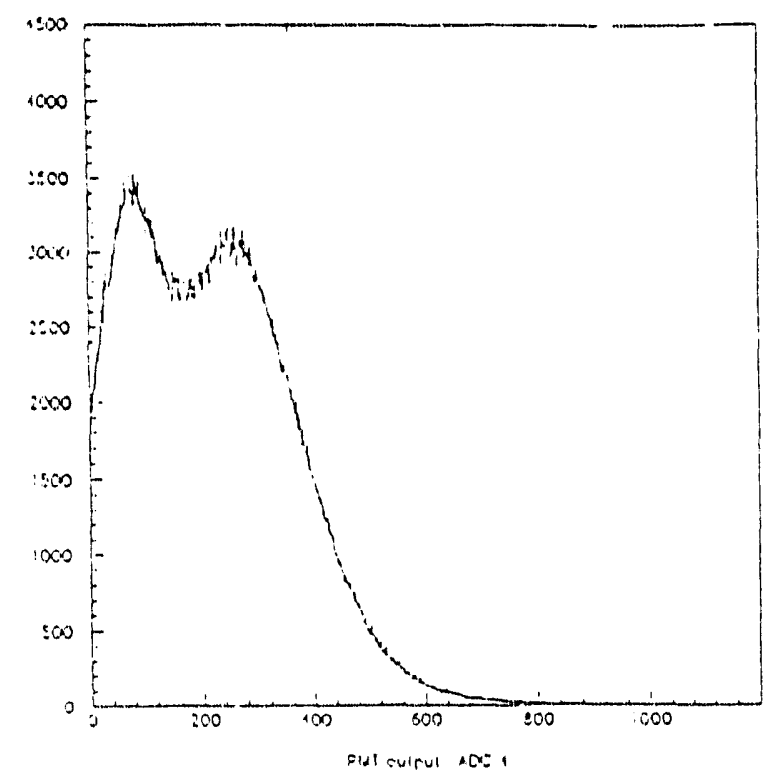

Figure 7: Single Photoelectron Peak and pedestal

the low light end, the signal was amplified by a high speed preamplifier $(\times 10.1)$ and a PS.777 amplifier $(\times 50)$. At the high light end, the amplifiers were replaced by a series of calibrated attenuators. The signal was recorded by an LRS $2249 \mathrm{~W}$ ADC with a sensitivity of $0.25 \mathrm{pC} /$ channel. The departure from linearity which is observed at the high end of fig 6 , is due to current limitation caused by the size of the storage capacitor. At the low end, there is considerable electronic pickup from the laser, which makes background subtraction very difficult.

By turning off the laser and covering the tube entrance hole, the single photoelectron peak was observed. At $V_{p c}=-8 \mathrm{kV}, \mathrm{V}_{A P D}=2 \mathrm{kV}$, it is well separated from the noise (see fig 7). The signal corresponds to $0.103 \mathrm{pC} /$ photoelectron with $\sigma=48 \%$. At $V_{x c}=7 \mathrm{kV}, \sigma$ is only $33 \%$. Sigma is expected to be even smaller with lower reverse bias voltages, but we have yet to improve our labaratory setup to be able to see the single photoelectron peak at these lower gains.

The noise figure is defined as the ratio of output signal width $(\sigma /<\operatorname{signal}\rangle)$ to input signal width. The noise figure of the hybrid tube has been measured to be 1.4, which is lower than the value of 1.6 typical of conventional phototubes.

A scope trace of the single photoelectron pulse after preamplification is shown in fig 3. This should be compared to fig 9 which shows a scope trace from the high end of the linearity studies. The pulse (no amplification, $\times 5$ attenuation) corresponds to 160 volts into $50 \Omega$. It shows no evidence of saturation, still exhibiting a characteristic 20 ns risetime.

\section{CONCLUSIONS AND FUTURE DIRECTIONS}

We bave designed and built a hybrid phototube which exhibits the following features:

(1) Gain of $5 \times 10^{5}$

(2) Linear over 6 decades

(3) Sensitive to single photoelectrons

(4) Quantum efficiency of $20-30 \%$, depending on photocathode choice

(4) Resistance to near-axial magnetic fields

(5) $10 \%$ variation in gain over active area

(6) $<.1$ ns transit time jitter 


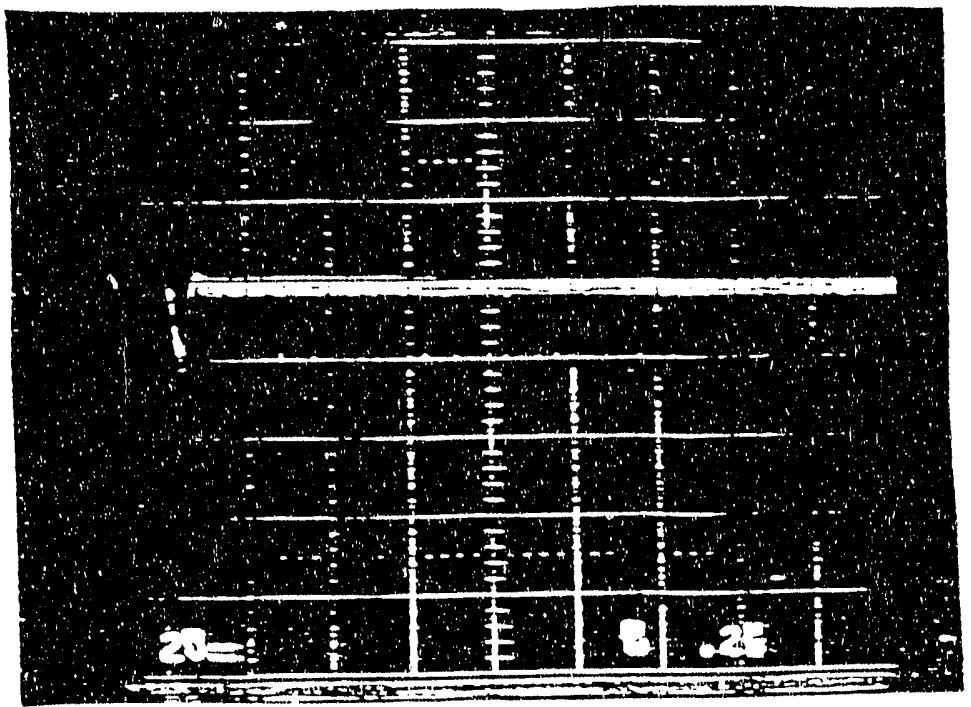

Figure 3: Scope trace of the single photoelectron signal with a $\times 10$ inverting preamplifier.

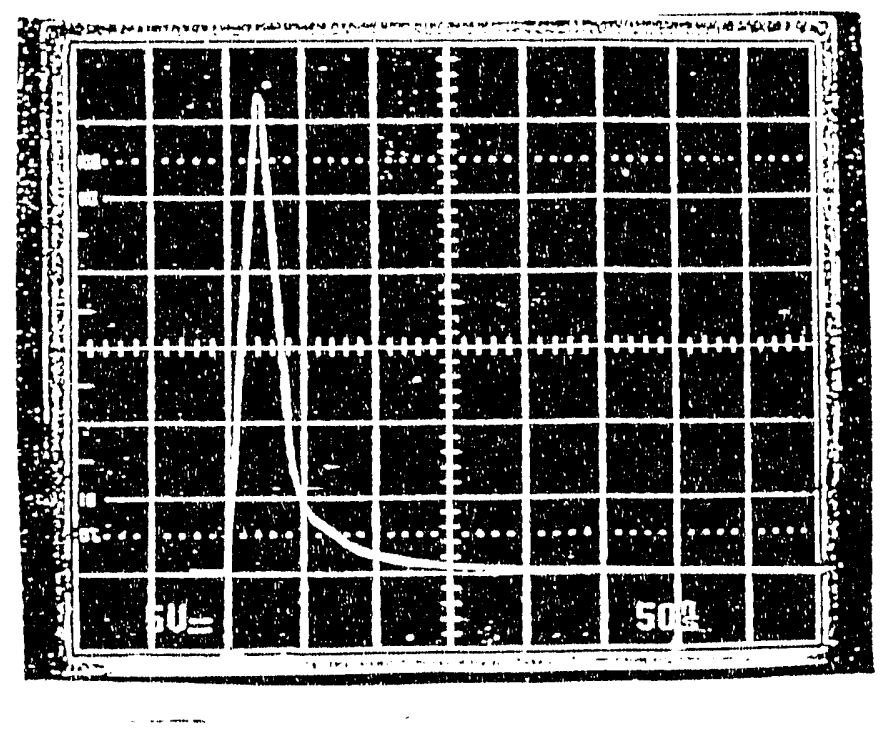

Figure 9: Scope trace of the signal at the high end of the linearity study summarized in fig 6 . The laser is operating without filters and a factor of 5 attenuator is on the scope input. The signal corresponds to 160 volts into $0 \Omega$, or 3.2 Amps peak output. 
(7) 20 ns risetime limited by area capacitance

(8) Simple, low current operation at room temperature

The lifetime of this tube has still to be quantitatively assessed, and we are in the process of doing such tests now. Electron bombardment could cause damage to the silicon itself, and attendant outgassing can poison the photocathode over time. All that can be said at this time is that after 4 months of testing, averaging 20 bours/week of "on" time over the whole range of input conditions, we have not observed any major damage.

We are interested in exploring its use as a readout for scintillating fibers, specificaily for the Shower Maximum Detector which is a finely segmented layer of the SDC tile/fiber calorimeter. To this end we will be subdividing the APD into an $8 \times 8$ array. The risetime for each channel will thus be several ns. In addition, the good surface uniformity implies that the channel-to-channel variation will be negligible. Crosstalk between channels is also expected to be $<1 \%$ since the tube is a simple planar accelerator. Axial magnetic fields would improve any vestigal crosstalk. The multi-anode version would also be very cost effective, an important consideration when designing readout for 40,000 channels. Initial estimates for 64 -channel devices in quantity, put the cost at $\$ 16.00 /$ channel.

\section{REFERENCES}

1. P.P.Webb, R.J.McIntyre, and J. Conradi, "Properties of Avalanche Photodiodes", RCA Review, Vol.35, p234 (1974).

2. R. Scharenberg, Private Communication.

\section{DISCLAIMER}

This report was prepared as an account of work sponsored by an agency of the United States Government. Neither the United States Government nor any agency thereof, nor any of their employees, makes any warranty, express or implied, or assumes any legal liability or responsibility for the accuracy, completeness, or usefulness of any information, apparatus, product, or process disclosed, or represents that its use would not infringe privately owned rights. Reference herein to any specific commercial product, process, or service by trade name, trademark, manufacturer, or otherwise does not necessarily constitute or imply its endorsement, recommendation, or favoring by the United States Government or any agency thereof. The views and opinions of authors expressed herein do not necessarily state or reflect those of the United States Government or any agency thereof. 

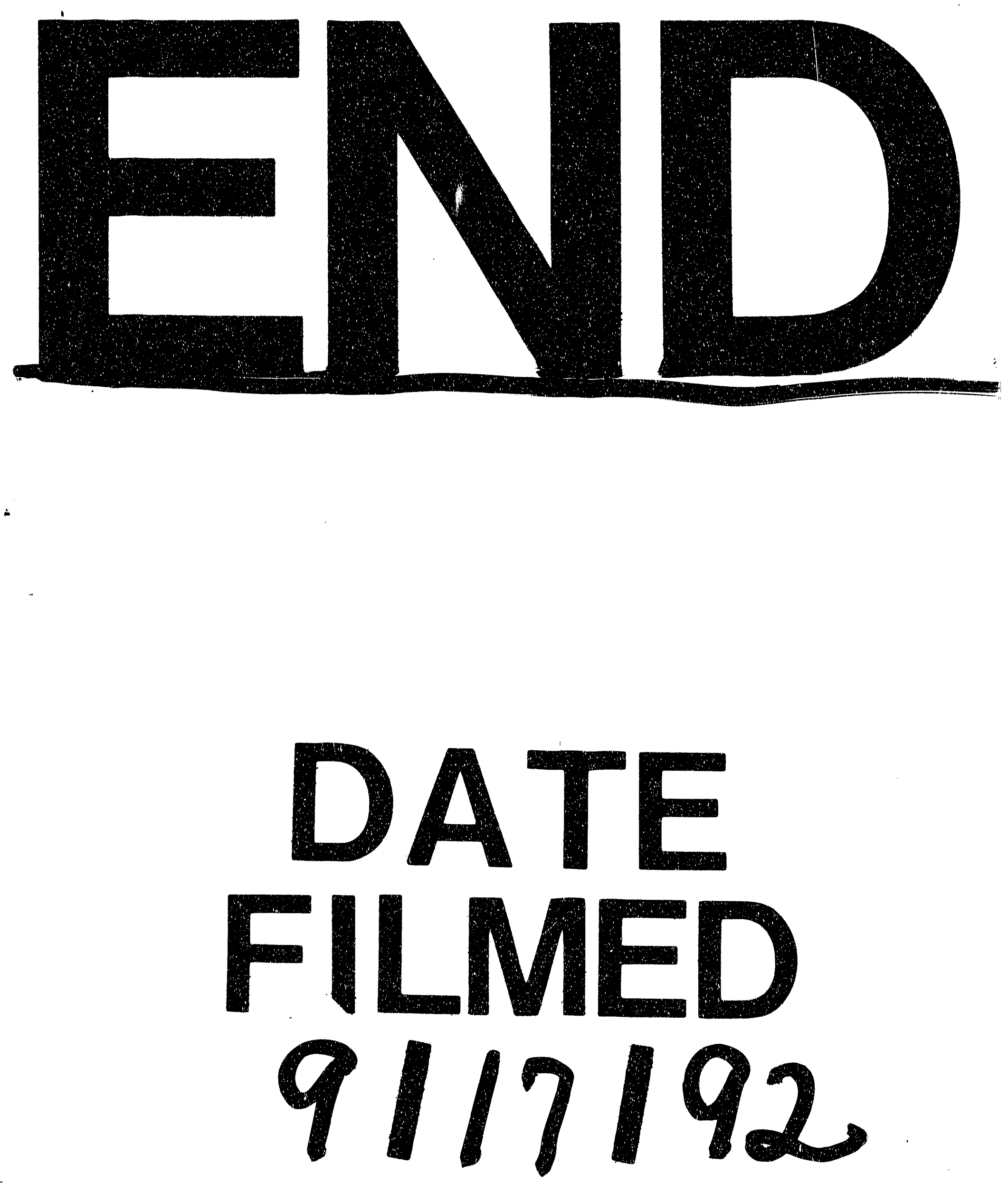
